\title{
EEG spectra during a long-term compensatory tracking task
}

\author{
CHARLES M. KORNFELD \\ Andrus Gerontology Center, University of Southern California, Los Angeles, California 90007
}

and

JACKSON BEATTY

University of California, Los Angeles, California 90024

\begin{abstract}
Seven subjects were tested in a prolonged (1-h 15 -min) compensatory manual tracking task with monopolar EEG (01, C3, and FP1) and EOG recorded. Five of the seven subjects displayed a performance decrement between the first and the fifth 15 -min periods of the task. For those five subjects, significant positive correlations were observed between performance and EEG activity at 01 in the 5.7- to 7.6- $\mathrm{Hz}$ frequency band. For the same subjects, performance was also inversely correlated with EEG activity at 01 in the high alpha band (9.6-11.5 Hz). Similar changes were observed in high-frequency activity at C3. A single significant correlation was observed between performance and EEG $(3.8-5.7 \mathrm{~Hz})$ at the frontal lead. This pattern is congruent with the hypothesis that EEG signs of activation are related to performance in a prolonged manual control task.
\end{abstract}

The study of human performance under monotonous conditions for prolonged periods of time has become a key paradigm for the study of sustained attention (Jerison, in press). In the typical vigilance task, signals are infrequently presented on a single display channel against a background of nonsignal events. Performance of the observer usually declines as a function of time in the task, a relationship termed the "vigilance decrement." Under such restrictions, behavioral efficiency is controlled by several principal factors including degree of signal predictability, observer motivation, and central nervous system activation or arousal.

The relations between central nervous system arousal and vigilance performance have become increasingly clear in the past decade. Electroencephalographic (EEG) indicators of cortical activation have been employed by several groups of investigators using vigilancetype tasks. With the exception of one unreplicated experiment reported by Daniel (1967), there is now convincing evidence that the vigilance decrement is accompanied by a decrease in cortical activation, as indexed by increases in theta frequency $(4-7 \mathrm{~Hz})$ activity in the posterior EEG. Theta activity in man is a sign of drowsiness and occurs most predominantly in Stage 1 sleep, a transitional state between sleep and waking.

The senior author, Charles Martin Kornfeld, died on 7 April 1976 at the age of 32 . This research was supported by the Defense Advanced Research Projects Agency under Contract N00014-70-C-0350 to Jackson Beatty. Additional on-line computer processing was provided by the Center for ComputerBased Behavioral Studies under Contract F30602-74-C-0016 with the Defense Advanced Research Projects Agency.
Beatty, Greenberg, Deibler, and O'Hanlon (1974) used a method of operant control of EEG in the theta band over the occipital hemisphere during a long radar watch in a successful attempt to affect a change in the efficiency of detection. Subjects who were conditioned to increase levels of theta activity showed an increased decrement in performance over time; subjects conditioned to decrease theta activity did not exhibit performance decrement. While using an auditory task, Davies and Krkovic (1965) observed decreased integrated alpha rhythm $(8-12 \mathrm{~Hz})$ with decreases in detection rate. Groll (1966) found a consistent rise in lowfrequency activity paralleling increases in reaction time (RT) in subjects instructed to respond to a light flash with a buttonpress. She examined both the frequency and amplitude of the occipital EEG in the $3 \mathrm{sec}$ preceding the presentation of a stimulus and found that the best predictor of the subject's RT was a negative relationship with the presence of theta frequency activity.

In each of the above studies, the behavioral index was a discrete discrimination task of low-probability stimuli. Such discrimination problems are the usual tasks for vigilance experiments. Discrete tasks of this sort lead to certain methodological problems in discerning whether the EEG shifts which vary with error are tied to general shifts in arousal, as defined by EEG frequency patterns, or to moment by moment shifts in the EEG preceding the presentation of a signal. For the study to be reported here, a slow compensatory tracking task was developed in which continuous behavioral data, as well as EEG data, could be recorded and complete correlational analyses performed. 


\section{METHOD}

Subjects were recruited for the experiment by advertisements posted in the psychology building at UCLA and advertisements placed in the University's daily paper. Persons inquiring were told that they would be required to be present in the laboratory on 2 consecutive mornings to perform a long and boring task. For this they would receive a maximum of $\$ 12$; in fact, all were paid the entire amount. If the prospective subject agreed to the above conditions, he or she was scheduled for the experiment. Seven right-handed subjects, five males and two females, between 20 and 28 years of age, participated.

The task was a random input compensatory tracking task, with each experimental session lasting $1 \mathrm{~h}$ and $15 \mathrm{~min}$. The subject was required to center a random moving dot on the horizontal axis of a cathode ray tube (CRT). The driving function (DF) was a smoothed $1 / 3-\mathrm{Hz}$ random signal. The driving function was added electronically to the subject's response signal (SR) from a freely moving control lever to form the displayed function, the compensatory tracking error (E). Six measures of tracking, the mean and root mean square (RMS) of the error, driving function and subject's response, were recorded during the experimental session by an ontine computer (Hewlett Packard 2116A).

Physiological recordings were taken on Day 2 only. All recordings were from silver-silver chloride electrodes, recorded on a Beckman R411 polygraph. The electro-oculogram (EOG) was recorded from electrodes placed at the right eye. The EOG was filtered with a high pass filter with a roll off frequency of $.16 \mathrm{~Hz}$, then digitized on-line and the mean and root mean squared voltage was calculated. Monopolar EEG was recorded from position $\mathrm{O} 1, \mathrm{C} 3$, and FP1 of the international 10-20 electrode system (Jasper, 1958), referenced to the right ear with the subject grounded at the left ear. The EEG was filtered at 1.6 and $30 \mathrm{~Hz}$ and recorded on a Hewlett Packard Model 3960A FM tape recorder.

Spectral analysis was performed on the EEG. The raw EEG data were first digitized, using an HP 2116A computer off-line. The digitized raw data were written on a magnetic tape, and the tape was transported to a Digital Equipment PDP-10 computer for spectral analysis. A fast Fourier transform (FFT) was then computed for the data in periods of $1,024 \mathrm{msec}$. The power spectral density was then calculated from the FFT, with the addition of the Hamming window technique to diminish the boxcar effect seen in a finite period spectral analysis. Percent power was then calculated for the following epochs: (1) 1.9$3.8 \mathrm{~Hz}$, (2) $3.8-5.7 \mathrm{~Hz}$, (3) $5.7-7.6 \mathrm{~Hz}$, (4) $7.6-9.6 \mathrm{~Hz}$, (5) $9.6-$ $11.5 \mathrm{~Hz}$, (6) $11.5-13.4 \mathrm{~Hz}$, (7) $13.4-20.1 \mathrm{~Hz}$.

For purposes of analysis, the task was divided into five consecutive 15 -min periods, with only data obtained in the first and last periods being fully analyzed.

\section{RESULTS AND DISCUSSION}

In this continuous tracking task, as in monotonous monitoring tasks, subjects showed a performance decrement as a function of time in task. Tracking performance was indexed by an error ratio (ERROR/ERROR + DRIVING FUNCTION), a statistic that approaches zero as performance improves and 1.0 as performance deteriorates. For the entire group of seven subjects, performance declined significantly between the initial and the final 15-min periods of the task $(t=3.078$, $6 \mathrm{df}, \mathrm{p}<.025)$. Analysis of the error data of individual subjects, however, revealed that five of the seven showed a significant performance decrement, whereas the remaining two did not. The behavioral data for the five

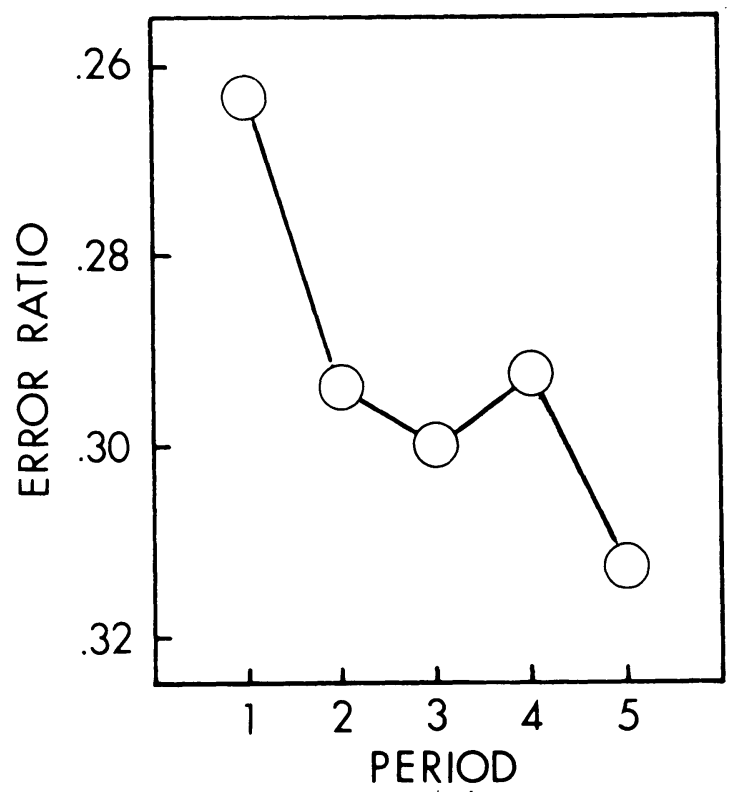

Figure 1. Mean error ratio averaged over the five subjects who showed a vigilance decrement for each of the five $15-\mathrm{min}$ periods of the task.

decrementing subjects are shown in Figure 1. No further analyses were performed on the two nondecrementing subjects.

The vigilance decrement observed in the compensatory tracking task was related to increased theta frequency activity, as has been previously reported for prolonged perceptual monitoring.

Table 1 contains the percent change values for each location and the correlations with the percent change in the error ratio. At the occipital lobe, significant positive correlations are seen for the 5.7 to $7.6 \mathrm{~Hz}$ bin. As the percent change in error ratio increases, the amount of high theta activity increases. Also, a negative correlation is seen in the high alpha range, 9.6 to $11.5 \mathrm{~Hz}$ bin. The central lead shows similar changes in the high-frequency activity. The effect in the low-frequency activity is not as strong. A single significant correlation was found at the frontal lead. There were no instances where highfrequency activity increased with error or low-frequency activity decreased with error.

The data were also analyzed to look for second by second fluctuation with error. The correlations calculated showed no pattern or epoch by epoch changes. All correlations were low.

In summary, it appears that the relationship between signs of low cortical activation, such as posterior theta frequency activity, and performance occurs not only in prolonged monitoring tasks, but in continuous compensatory tracking tasks as well. The continuous tracking task, we believe, offers some unique methodological advantages for the study of sustained attention. First, a performance index is continuously available in the tracking task, enabling the detailed analysis of the fine 
Table 1

EEG Data Percent Change

\begin{tabular}{|c|c|c|c|c|c|c|c|c|}
\hline & $\begin{array}{l}\text { Error } \\
\text { Ratio } \\
\end{array}$ & $\begin{array}{c}1.9-\text { to } \\
3.8-\mathrm{Hz} \\
\mathrm{Bin}\end{array}$ & $\begin{array}{c}3.8-\text { to } \\
5.7-\mathrm{Hz} \\
\mathrm{Bin}\end{array}$ & $\begin{array}{c}5.7-\text { to } \\
7.6-\mathrm{Hz} \\
\mathrm{Bin}\end{array}$ & $\begin{array}{c}7.6-\text { to } \\
9.6-\mathrm{Hz} \\
\mathrm{Bin}\end{array}$ & $\begin{array}{c}9.6-\text { to } \\
11.5-\mathrm{Hz} \\
\text { Bin }\end{array}$ & $\begin{array}{c}11.5-\text { to } \\
13.4-\mathrm{Hz} \\
\text { Bin }\end{array}$ & $\begin{array}{c}13.4-\text { to } \\
20.1-\mathrm{Hz} \\
\text { Bin }\end{array}$ \\
\hline & \multicolumn{8}{|c|}{ Occipital Lead } \\
\hline Subject 1 & 26.6 & -47.9 & 56.2 & 131.1 & 66.8 & -23.2 & 64.0 & -12.7 \\
\hline Subject 2 & 22.1 & 5.8 & 3.9 & 1.0 & -1.2 & -2.6 & -.6 & -5.8 \\
\hline Subject 3 & 12.9 & -62.1 & 17.7 & 44.6 & 74.2 & -23.0 & -12.5 & -34.6 \\
\hline Subject 4 & 9.5 & -13.0 & 95.6 & -5.9 & -42.8 & 24.5 & -23.9 & -45.0 \\
\hline Subject 5 & -1.5 & -46.2 & -75.6 & -49.5 & 27.7 & 88.6 & 73.1 & -23.4 \\
\hline Correlation & & .221 & .520 & .800 & .184 & -.859 & -.210 & .551 \\
\hline $\mathrm{t}$ Value & & .392 & 1.054 & 2.304 & .324 & -2.906 & -.372 & 1.144 \\
\hline \multirow[t]{2}{*}{$\mathrm{df}=4, \mathrm{p}<$} & & n.s. & n.s. & .05 & n.s. & .025 & n.s. & n.s. \\
\hline & \multicolumn{8}{|c|}{ Central Lead } \\
\hline Subject 1 & 26.6 & 5.36 & 51.8 & 37.8 & -13.5 & -43.0 & -56.1 & -50.7 \\
\hline Subject 2 & 22.1 & 9.1 & 4.2 & 8.6 & -9.5 & -3.6 & 5.6 & 1.7 \\
\hline Subject 3 & 12.9 & -16.0 & 39.4 & -27.4 & 11.5 & -10.3 & -42.9 & 125.8 \\
\hline Subject 4 & 9.5 & -59.0 & 104.5 & 71.5 & -22.5 & -8.1 & 17.3 & -9.2 \\
\hline Subject 5 & -1.5 & -29.2 & -53.4 & 32.1 & 31.5 & 34.1 & 26.1 & -39.6 \\
\hline Correlation & & .710 & .392 & -.144 & -.673 & -.872 & -.833 & -.034 \\
\hline t Value & & 1.746 & .738 & -.252 & -1.570 & -3.085 & -2.608 & -.054 \\
\hline \multirow[t]{2}{*}{$\mathrm{df}=4, \mathrm{p}<$} & & .10 & n.s. & n.s. & .10 & .025 & .05 & n.s. \\
\hline & \multicolumn{8}{|c|}{ Frontal Lead } \\
\hline Subject 1 & 26.6 & 122.9 & 74.3 & -58.1 & -75.0 & -67.4 & -69.8 & -90.05 \\
\hline Subject 2 & 22.1 & 1.7 & 3.9 & 6.3 & -4.9 & -3.8 & 5.5 & -3.2 \\
\hline Subject 3 & 12.9 & -45.7 & 24.1 & -32.7 & 86.2 & 25.5 & -18.6 & .4 \\
\hline Subject 4 & 9.5 & -15.6 & -20.4 & -5.0 & 72.0 & 10.1 & 1.7 & 118.7 \\
\hline Subject 5 & -1.5 & 58.6 & -23.7 & -40.8 & 21.3 & -26.5 & -35.0 & -51.5 \\
\hline Correlation & & .266 & .796 & -.001 & -.605 & -.343 & -.213 & -.259 \\
\hline t Value & & .478 & 2.278 & -.002 & -1.316 & -.632 & -.378 & -.464 \\
\hline $\mathrm{df}=4, \mathrm{p}<$ & & n.s. & .05 & n.s. & n.s. & n.s. & n.s. & n.s. \\
\hline
\end{tabular}

temporal structure relating brain state and performance. Second, the measure of performance is graded rather than binary, which permits detailed behavioral analysis.

Finally, it should be mentioned that the continuous tracking task employed in this study is analogous in many ways to some operational control tasks that occur in applied settings. Compensatory tracking of a slowly changing error forcing function may serve as a laboratory model for vehicle control, for example. Recent evidence (O'Hanlon \& Kelley, in press) has suggested that vigilance-type decrements may be a chronic occupational hazard in long-haul truck driving. The laboratory study of continuous control for prolonged periods might provide insights into the brain/behavior relationships that operate in such situations.

\section{REFERENCES}

Beatty, J., Greenberg, A., Deibler, W. P., \& O'Hanlon, J. F. Operant control of occipital theta rhythm affects performance in a radar monitoring task. Science, 1974, 183, 871-873.

DAniel, R. S. Alpha and theta EEG in vigilance. Perceptual and Motor Skills, 1967, 25, 697-703.

Davies, D. R., \& KRKovic, A. Skin conductance, alpha activity and vigilance. American Journal of Psychology, 1965, 78, 304-306.

Groll, E. Central nervous system and peripheral activation variables during vigilance performance. Zeitschrift für Experimentelle und Augewandte Psychologie, 1966, 13, 248-264.

JASPER, H. H. The ten-twenty electrode system of the International Federation. Electroencephalography and Clinical Neurophysiology, 1958, 10, 371-375.

JERISON, H. J. Vigilance: Biology, psychology, theory and practice. In R. R. Mackie (Ed.), Vigilance II. New York: Plenum, in press.

O'Hanlon, J. F., \& Kelley, G. R. Comparison of CNS and autonomic changes between drivers who perform well and poorly during prolonged vehicular operation. In $\mathbf{R} . \mathbf{R}$. Mackie (Ed.), Vigilance II. New York: Plenum, in press.

(Received for publication March 3, 1977.) 